\title{
Mode Suppression Ratio of CW Laser Diode and its Influence on Optical Communication Network
}

\author{
Dr. Erada A. K. Al-Dabbagh \\ Department of Physics \\ College of Science \\ University of mosul
}

Received
$10 / 01 / 2011$

\author{
Mohib Aldeen M. Hayawe \\ Department of Physics \\ College of Education \\ University of mosul
}

\section{Accepted}

12 / 04 / 2011

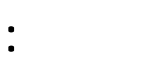

مم في هذا البهث درلسة تأثير نسبة إخماد النط على دينميكية ليزرات أشبها

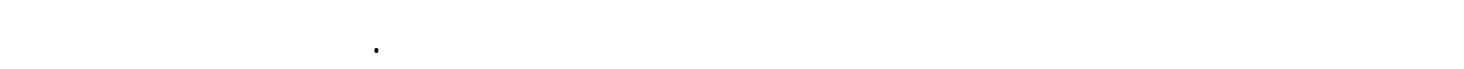
ضوئية عند لف ضوئي طول 5m k0، وتحمل ليزرشبه موصل عند 10 Gbps. القيمة

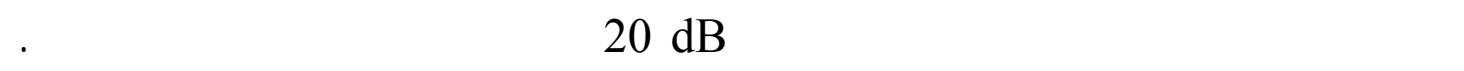

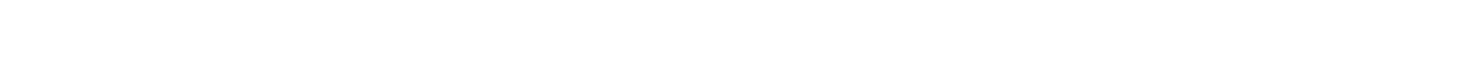

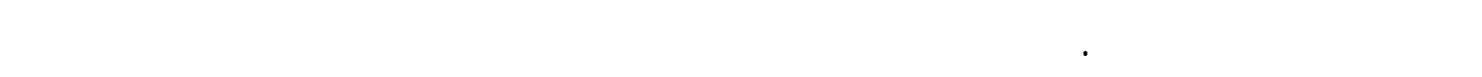

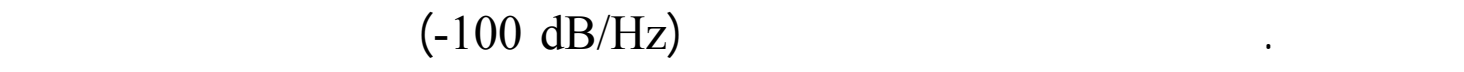

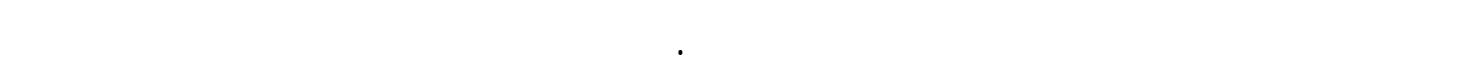
المخطط الضوئي عنما لخذت الزحزحة بشظر الاعتبار.

\section{Abstract:}

The influence of mode suppression ratio on the dynamics of semiconductor laser will be investigated in this paper, especially in a high bit rate optical communication. An optical communication network of fiber length of $50 \mathrm{~km}$ was established, with semiconductor laser modulated at $10(\mathrm{Gbps})$. Mode suppression ratio of more than $20 \mathrm{~dB}$ was an optimum value for a laser diode to be considered as a single mode laser. We have taken into account in this study the following factors namely; the number of longitudinal modes, the relative intensity noise, laser mode linewidth, and chirping. The result showed that, the quality factor and bit error rates were affected by the mode suppression ratio. Relative intensity noise of $(-100 \mathrm{~dB} / \mathrm{Hz})$ had an extreme drop in the 
received power and more fluctuations were found in the power output spectra. Intramodal distortion was observed in the optical layout system when chirping was considered in the laser characteristic.

\section{1- Introduction:}

Optical communication network usually works with laser diode as a transmitter in the wavelength $1550 \mathrm{~nm}$ where the fiber loss is in its minimum. Laser diode spectral linewidth will play an important role in its characteristics by bandwidth and bit rate[1]. Laser phase noise and relative intensity noise are restricting the bit rate and BER (bit error rate)[2]. Phase stability of laser diode and its influence on linewidth had been pointed out by Gliese et.al and a stable requirement for coherent optical beam characteristics of the laser diode[3]. Spectral purity of a very narrow linewidth laser transmitter allows the phase modulation of data signals had bit rate as low as a $155 \mathrm{Mb} / \mathrm{s}$, which alleviates the need for digital signal processing in the detection circuit[4]. Laser diode injection current is another noise component and the tuning mechanism for direct modulation will convert this drive current noise as an additional frequency fluctuations "frequency jitter" components, so all noise components mentioned will contribute to the measured linewidth[5]. Mode-beating exhibits a narrow linewidth below $100 \mathrm{kHz}$, and low jitter components with high frequency correlation between these modes [6].

It has shown that amplified spontaneous emission can affect laser diode linewidth and relative intensity noise (RIN), and the enhancement linewidth factor [7]. Recently, it has been shown that for long haul optical communication system, single mode laser diode is the most cost effective transmitter due to the limited chromatic dispersion and polarization mode dispersion [8]. On the other hand, side mode rejection ratio (MSR) has influence on the coherency of laser diode [9].

\section{2- Theory:}

In semiconductor lasers, the linewidth enhancement factor $(\boldsymbol{\alpha})$ plays a great role in deterring its spectral linewidth. This factor can be defined as [9]:

$$
\alpha=\frac{\operatorname{Re}[\chi]}{\operatorname{Im}[\chi]}=-2 \frac{\omega}{c} \frac{\frac{\partial \eta}{\partial n}}{\frac{\partial g}{\partial n}}
$$

Where $\chi$ is the complex electric susceptibility, $\boldsymbol{\eta}$ is the refractrive index, $\boldsymbol{n}$ is the carrier density, $g$ is the gain, $\boldsymbol{\omega}$ is the angular frequency of the laser, and $\boldsymbol{c}$ is the speed of light.

The spectral linewidth is given, taking into account the $\alpha$ parameter, as [9]:

$$
\Delta v=\frac{R_{s p}}{4 \pi p_{h}}\left(1+\alpha^{2}\right)
$$


with $\boldsymbol{R}_{\boldsymbol{s} p}$ is the spontaneous emission factor that emitted with laser output contributing as a noise in the laser output, and $p_{h}$ is the photon number.

When the laser diode is directly modulated with angular frequency $\omega$, a rich harmonics and relative intensity noise were observed. This modulation power spectrum has a resonance peak around the realxation oscillation $\omega_{R}$. The influence of both $\boldsymbol{R}_{s p}$ and $\boldsymbol{\alpha}$ on the power spectrum of the phase noise can be seen from the following equation for the phase noise[ 9 ]:

$\phi_{\Delta \omega}(\omega)=\frac{R_{s p}}{2 p_{h}}\left\{1+\frac{\alpha^{2} \omega_{R}^{4}}{\left(\omega_{R}^{2}-\omega^{2}\right)^{2}+\left(2 \omega \Gamma_{R}\right)^{2}}\right\}$

Knowing that $\boldsymbol{\Gamma}_{R}$ is the damping factor of the relaxation oscillations [9].

Hence, modulation characteristics of the laser diode is affected by many factors, such as spectral linewidth $\Delta v$, the spontaneous emission factor $\boldsymbol{R}_{s p}$ and the linewidth enhancement factor $\boldsymbol{\alpha}$.

The modulation characteristics of any laser diode is also affected by the number of longitudinal modes and the side-mode suppression ratio (SMSR) [10]. The optical field of the laser output, taking into account these effects, can be written as [11]:

$E_{\text {out }}(t)=\sqrt{P}\left[1+s \cdot \cos \left(2 \pi \Delta_{f} t\right)+s \cdot \cos \left(-2 \pi \Delta_{f} t\right) e^{i \varphi}\right.$

where $\boldsymbol{P}$ is the laser output power, $\boldsymbol{s}$ is the supression ratio, and $\Delta_{f}$ is defined as the separation parametetr.

If the parameter independent side mode is enabled, the average signal power will be greater than $\boldsymbol{P}$, since it includes the contribution from the side mode.If this parameter is disabled, the output power will be $\boldsymbol{P}$. This means that, the signal will be scaled in order to give the same average power. The signal phase and polarization is calculated in the same way as the $\mathrm{CW}$ laser.

The model can also works as a Fabry-Perot laser; in this case, the parameter number of side modes defines the number of modes of the laser. The normalized power for each mode is calculated based on the power of the central mode and the power of the first side mode [12], according to:

$P_{n}=\frac{1}{1+\left(\frac{1}{P_{s}}-1\right)\left(\frac{\eta_{1}}{m}\right)^{2}}$

Where $\boldsymbol{m}$ is the number of side modes, $\eta_{1}$ is the index of each side mode pair, and $\boldsymbol{P}_{\boldsymbol{s}}$ is calculated from the power (number of photons) of the first side mode:

$P_{s}=\frac{1}{\left(\frac{1}{s}-1\right) m^{2}+1}$ 
When RIN is enable, the model generates noise with bandwidth and spacing that you define. The parameter RIN is the ratio of the meansquare optical intensity noise to the square of the average power [12][13]: $\mathrm{RIN}=\frac{\left\langle\Delta \boldsymbol{P}^{2}\right\rangle}{\boldsymbol{P}_{m}^{2}} \quad \boldsymbol{d B} / \mathrm{Hz}$

Where $\Delta \boldsymbol{p}^{2}$ is the mean-square optical intensity fluctuation at a specific frequency and $\boldsymbol{p}_{m}^{2}$ is the measured power. These models estimates $\Delta \boldsymbol{p}^{2}$ based on the parameters RIN and measured power.

The signal phase and polarization is calculated in the same way as the $\mathrm{CW}$ laser, where the laser phase noise is modeled using a Gaussian random variable for the phase difference between two successive time instants with zero mean and a variance equal to $2 \pi \sqrt{\Delta \mathbf{v}}$, where $\Delta \boldsymbol{v}$ is the laser Linewidth.

Chirping occurs when the carrier density in the active region is rapidly changed, causing a shift of the index of refraction with time. This modifies the frequency of the output light (the term "chirp" refers to a linear change in frequency over time) and affects dispersion and other link noise sources [14]. Chirping is most often observed in pulsed sources. The chirping effect on the transmitted power through the optical fiber will be considered implicitly in the optical network layout. The chirp to the power ratio can be described in the following equation [15]:

$$
C P R=\frac{1}{2 \pi R_{p}}\left|\frac{d \varphi}{d t}\right|
$$

Where $\boldsymbol{R}_{\boldsymbol{p}}$ is the modulation response of the laser diode with respect to the modulation frequency, and $\frac{d \varphi}{d t}$ represents the change in the phase of laser cavity due to chirping.

\section{3- Optical Communication Layout:}

The optical network that has been proposed in this work will be using the state-of-the-art of high bit rate $(10 \mathrm{GHz})$ laser diode as a transmitter. Fig. (1) shows the proposed network layout. The bit sequence was generated from a pseudo-random unit and connected to non-return-tozero (NRZ) pulse generator. The laser diode unit had been selected from the menu of the computer aided design package (Optisystem 7.0) with rate equations characteristics that can be varied through the simulations. Laser diode rate equations have many parameters that must be given in order to simulate the layout, including carrier and photon lifetimes, bias current \& modulation currents, and wavelength, as given by Table (1).

The table represents the values of the laser parameters that have been used through the simulation. The single mode laser or multimode laser can be characterized by defining the number of modes, side mode 
rejection ratio and laser linewidth. The single mode laser in the simulation had a linewidth of $10 \mathrm{MHz}$ with no side mode. While the multimode laser had a linewidth of $100 \mathrm{MHz}$ and (6) side modes.

The optical fiber parameters are given in Table (2). These parameter values will be used in the simulation for the optical network layout.

Table (1): Laser Diode Parameters and their Values

\begin{tabular}{|c|c|c|}
\hline Name of Parameter & Value & Units \\
\hline Laser wavelength & 1.552 & $\mathrm{~mW}$ \\
\hline Laser power output & 2 & $\mathrm{~dB} / \mathrm{Hz}$ \\
\hline Number of Side mode & 6 & $\mathrm{~mA}$ \\
\hline Relative intensity noise & -130 & $\mathrm{~W} / \mathrm{A}$ \\
\hline Threshold current & 20 & \\
\hline Slope efficiency & 0.6 & \\
\hline Linewidth & variable & \\
\hline Mode suppression ratio & variable & \\
\hline
\end{tabular}

Table (2): Optical Fiber Parameters and their Values at Laser Wavelength $1.552 \mu \mathrm{m}$. Fiber Length was Taken as $50 \mathrm{~km}$ as a Moderate Length for Short and Long Haul Distance for Optical Communication Network.

\begin{tabular}{|c|c|c|}
\hline Name of parameter & Value & Units \\
\hline Length & 50 & $\mathrm{~km}$ \\
\hline Attenuation & 0.2 & $\mathrm{~dB} / \mathrm{km}$ \\
\hline Dispersion & 8 & $\mathrm{ps} / \mathrm{nm} / \mathrm{km}$ \\
\hline Effective area & 80 & $\mu \mathrm{m}$ \\
\hline Differential group delay & 0.2 & $\mathrm{ps} / \mathrm{km}$ \\
\hline
\end{tabular}




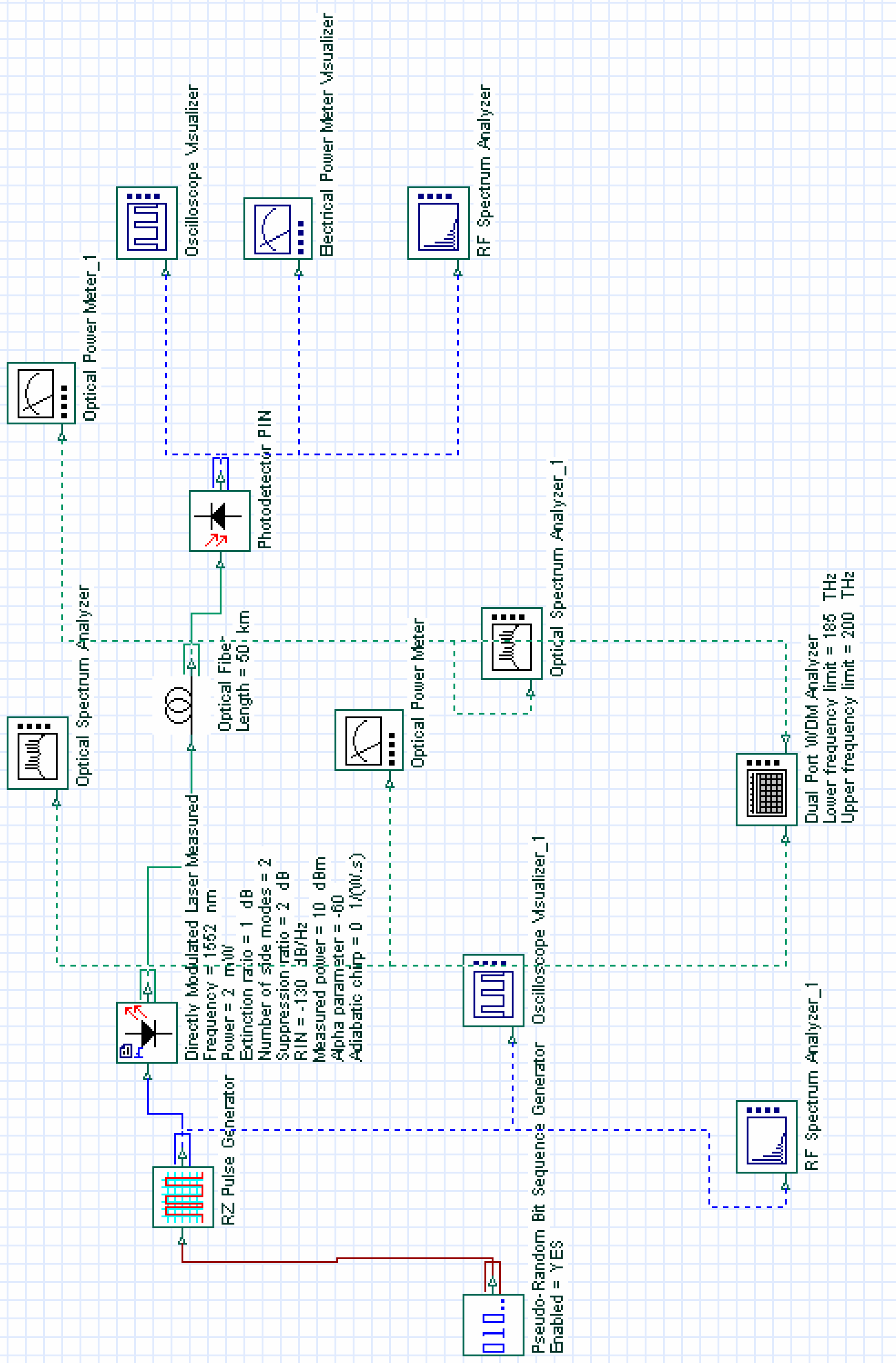

Fig. (1): Optical communication layout 


\section{4- Results and discussion:}

In order to study the effect of number of modes on the laser spectrum, Fig. ( $2 a \& b)$ shows the spectra of the laser diode for single and multimode laser. Fig. (2a) shows the spectrum of single mode laser with linewidth of $10 \mathrm{MHz}$ and power output $15 \mathrm{~mW}$. From the figure, we can see that the laser is completely a single mode, and with side mode rejection ratio is more than $(13.3 \mathrm{~dB})$. On the other hand, when the mode suppression ratio is low, such as $(4.9 \mathrm{~dB})$, a multimode spectrum will be observed, as shown in Fig. (2b).
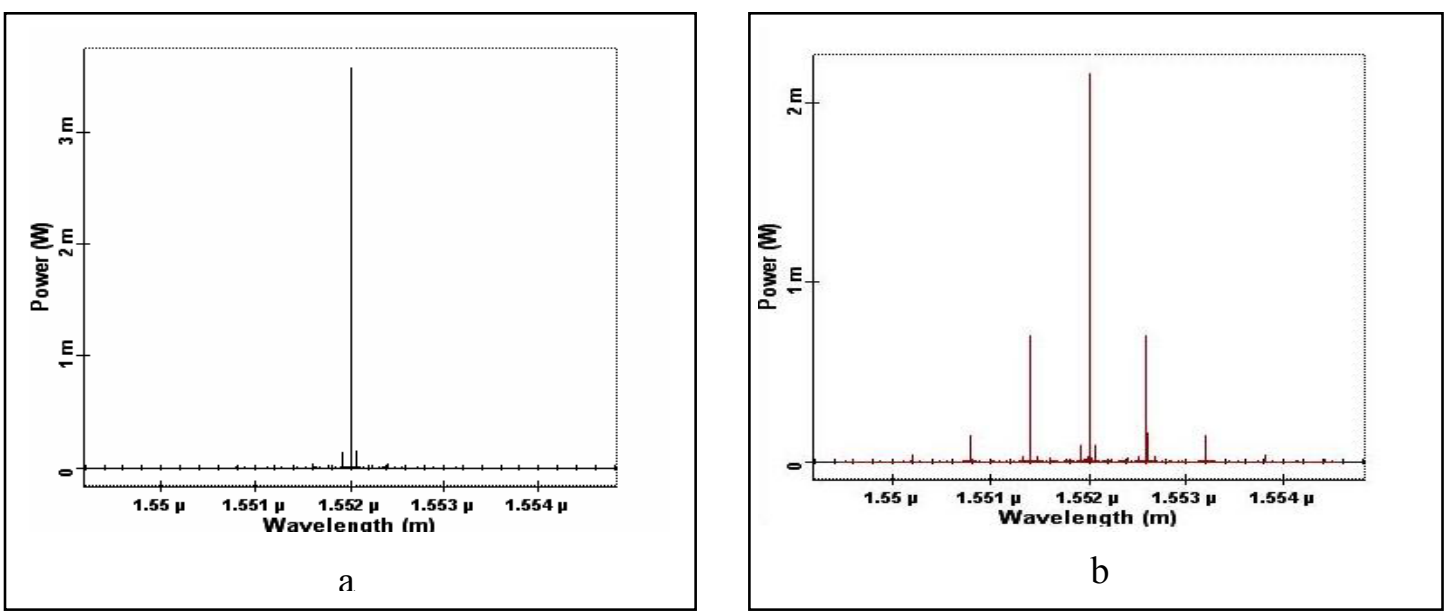

Fig. (2a\&b): Spectrum of the laser diode (a) single mode (b) multi mode.

As a matter of fact, the power in the main mode should be as large as ten times more than the power of the nearest mode [11]. This will be used throughout the simulation in order to fulfill this requirement.

Fig. (3a\&b) illustrates the influence of launching such a spectrum (single mode $\&$ multimode) with a fiber length $(50 \mathrm{~km})$. Due to the fiber effects, single mode laser spectrum (Fig. (3a)) had decreased in its power output. While the decrease in the power output of the multimode spectrum of the laser diode emerging from the fiber had a decrease in the main mode and the side modes as well, as shown in Fig. (3b), which gives less coherency and less side mode rejection ratio.
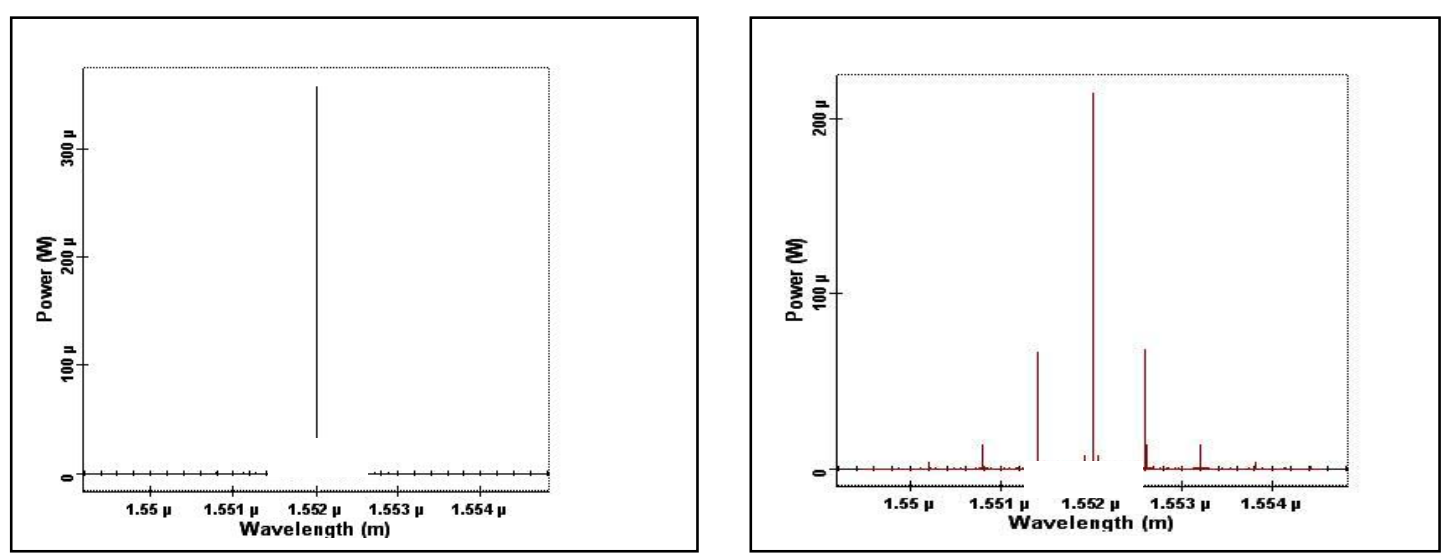

Fig. (3a\&b) The influence of launching a spectrum (a) single 
The influence of mode suppression ratio (MSR) on the quality factor (Q.F) and Bit Error Rate (BER) of the optical network will be discussed. The quality factor was zero at MSR equal to $5 \mathrm{~dB}$, and Q.F was increased to 5.5 at MSR of $25 \mathrm{~dB}$. The BER at MSR of $25 \mathrm{~dB}$ became $\left(1.6 \times 10^{-8}\right)$. These values were obtained when the eye diagram of the receiving signal from the photodiode was analyzed, so eye diagram analysis will be discussed.

Usually any optical network analyzed its performance through the study of eye diagram. Eye diagram of a single mode laser diode is shown in Fig. (4a) while the eye diagram of the multimode laser diode is shown in Fig. (4b).
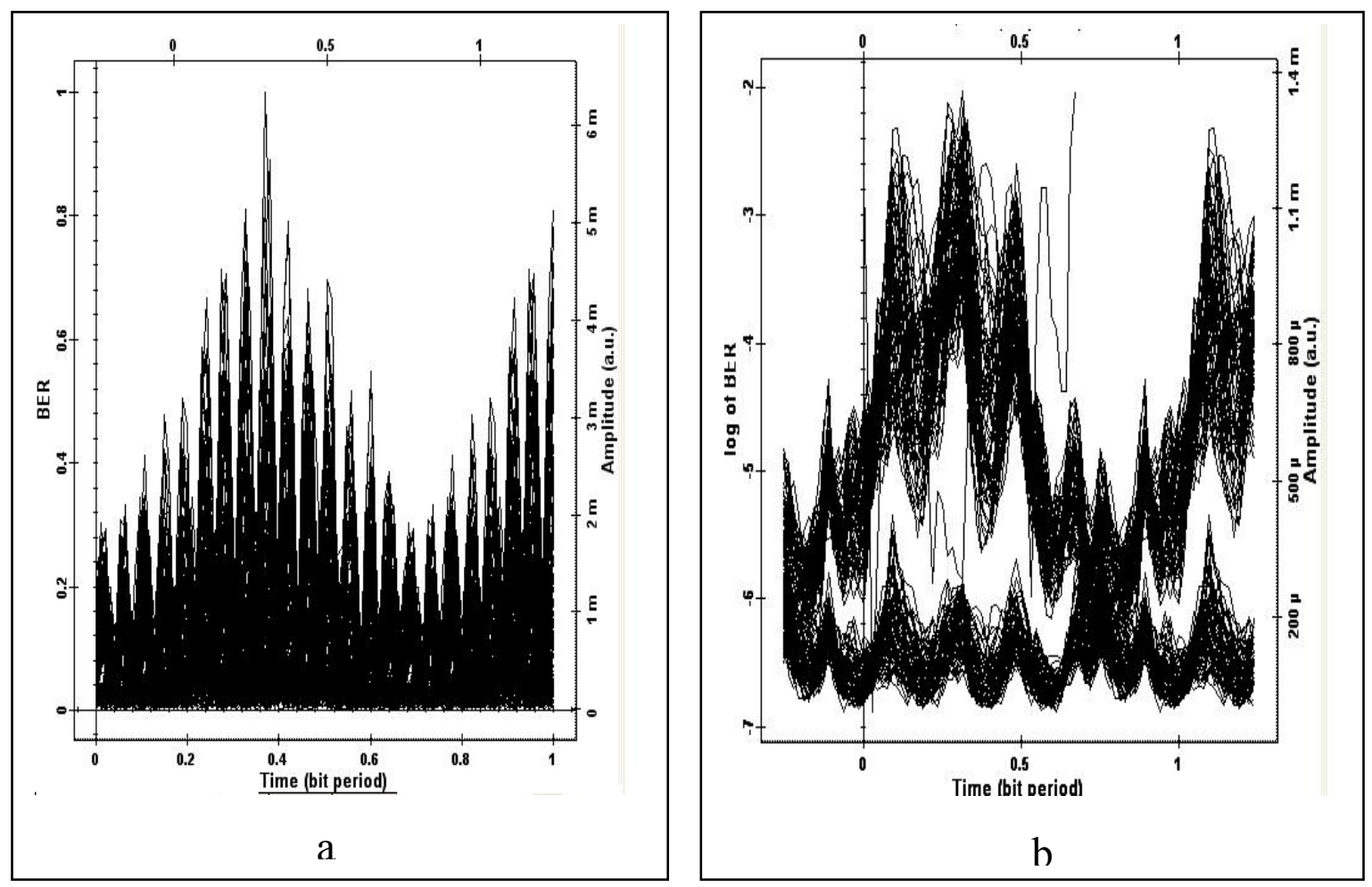

Fig. (4a\&b): Eye diagram of a (a) single mode laser diode (b) multimode laser diode.

As can be seen from Fig. (4a), the bits can be recognized at a bit rate of $10 \mathrm{GHz}$ with high amplitude and low BER. This diagram show a pronounced peaks for the receiving signal. Fig. (4b) shows the eye diagram for the multi mode laser with a high BER, low amplitude and overlapping between the peaks that will indicate a very low performance for the network.

The idea of single and multimode laser diode as a transmitter of 10 Gbps data rate and its effects on the optical communication network for different fiber length will be presented. Fig. (5) shows the attenuation in the power output as a function of fiber length for both single mode and multimode laser. As can be seen from the figure that the power of the receiving signal is more decreased in multimode than single mode. 


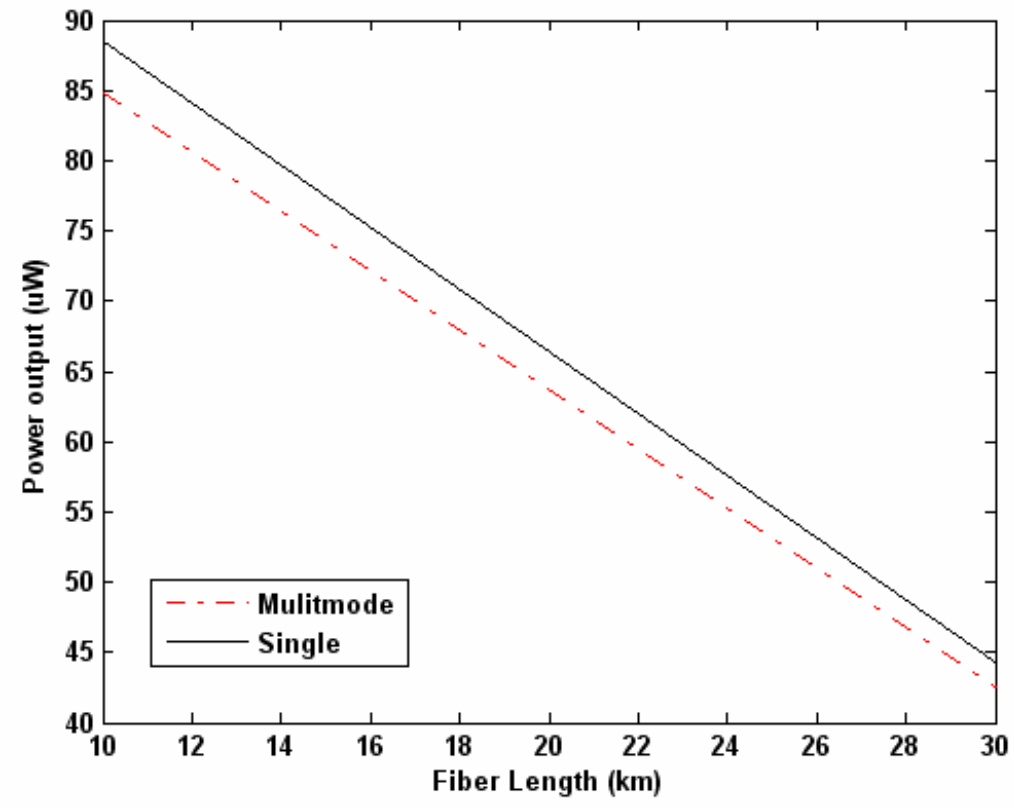

Fig. (5): Single and multimode laser diode as a transmitter and its effects on the optical communication network.

This study will consider the effect of the relative intensity noise (RIN) on the proposed optical network. The influence of the RIN is on the normalized power output when launched through a fiber length of 50 $\mathrm{km}$ as shown in Fig.(6) for two values of $(\mathrm{RIN}=-130 \&-100 \mathrm{~dB} / \mathrm{Hz})$. The extreme drop of the power was observed when the RIN was equal to -100 $\mathrm{dB}$ more than when RIN was $=-130 \mathrm{~dB} / \mathrm{Hz}$. This can be explained (with the aid of Eq. (7)) due to the more fluctuation in the power of the laser diode when RIN was increased.

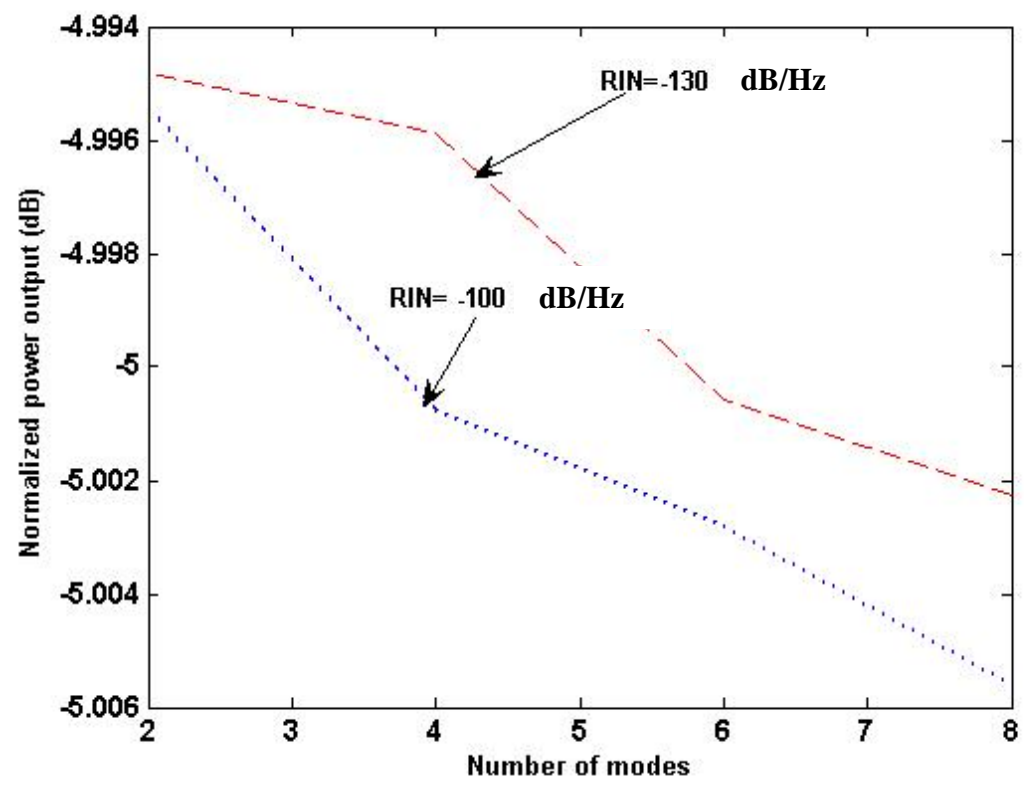

Fig. (6): The normalized power output as a function of number of modes for two different values of RIN. The MSR $=2 \mathrm{~dB}$, laser linewidth $=20 \mathrm{MHz}$ and fiber length $=50 \mathrm{~km}$. 
Chirping effect on the proposed optical network had been taken into account, as a parametric value in the laser diode characteristic when externally direct modulated. This effect can be observed on the normalized power output as a function of number of modes as shown in Fig. (7). The influence of chirping on the power output is fluctuating at different mode number. This effect has been recognized as a partition noise or mode partition noise. In an optical communication link, mode partition noise is a phase jitter of the signal caused by the combined effects of mode hopping in the optical source and intramodal distortion in the fiber.

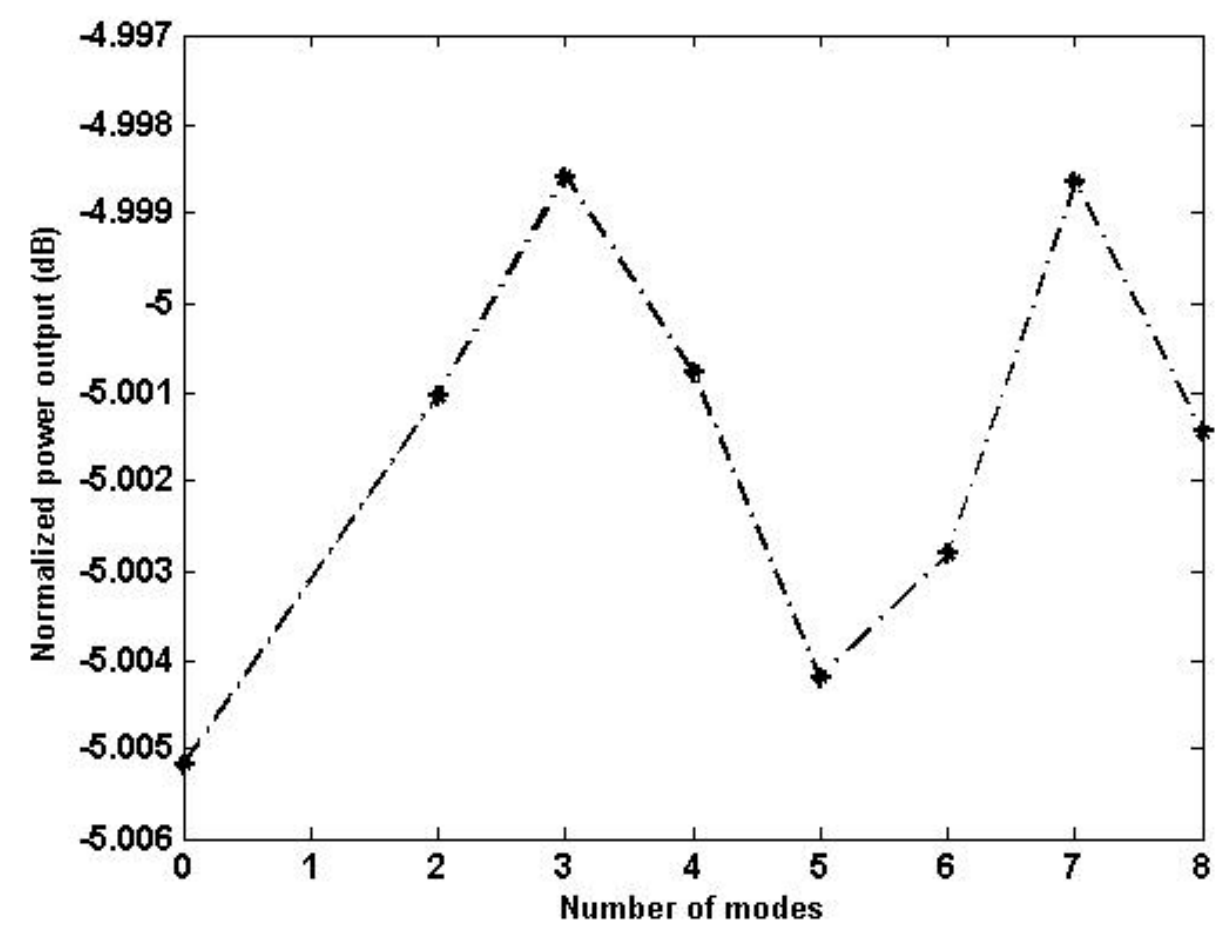

Fig.(7): The normalized power output as a function of number of mode with chirping, at modulation frequency $10 \mathrm{GHz}, 50 \mathrm{Km}$ fiber length , MSR=2 dB, $\mathrm{RIN}=-130 \mathrm{~dB} / \mathrm{Hz}$, and laser linewidth $=20 \mathrm{MHz}$.

To summarize the results as a comparison between the performance of single mode laser and multimode laser in our proposal of optical communication layout, Table (3) summarize these results. As one can conclude that, for $50 \mathrm{~km}$ network, multimode laser showed a poor performance. This suggests that multimode laser is no longer fit for more than $(10-20 \mathrm{~km})$ fiber length.

Table (3): Comparison between single mode and multimode laser performance

\begin{tabular}{|c|c|c|}
\hline Parameter & Single Mode & Multimode \\
\hline MSR & $\geq 20 \mathrm{~dB}$ & $\leq 5 \mathrm{~dB}$ \\
\hline RIN & $-130 \mathrm{~dB} / \mathrm{Hz}$ & $-100 \mathrm{~dB} / \mathrm{Hz}$ \\
\hline BER & $1.6 \times 10^{-8}$ & $10^{-2}$ \\
\hline Q.F. & 5.5 & 0 \\
\hline
\end{tabular}


In order to show how multimode laser can signifies the nearest side mode to the dominant mode compared to single mode laser, we had solved Eqn. (5) numerically as shown in Fig. (8).

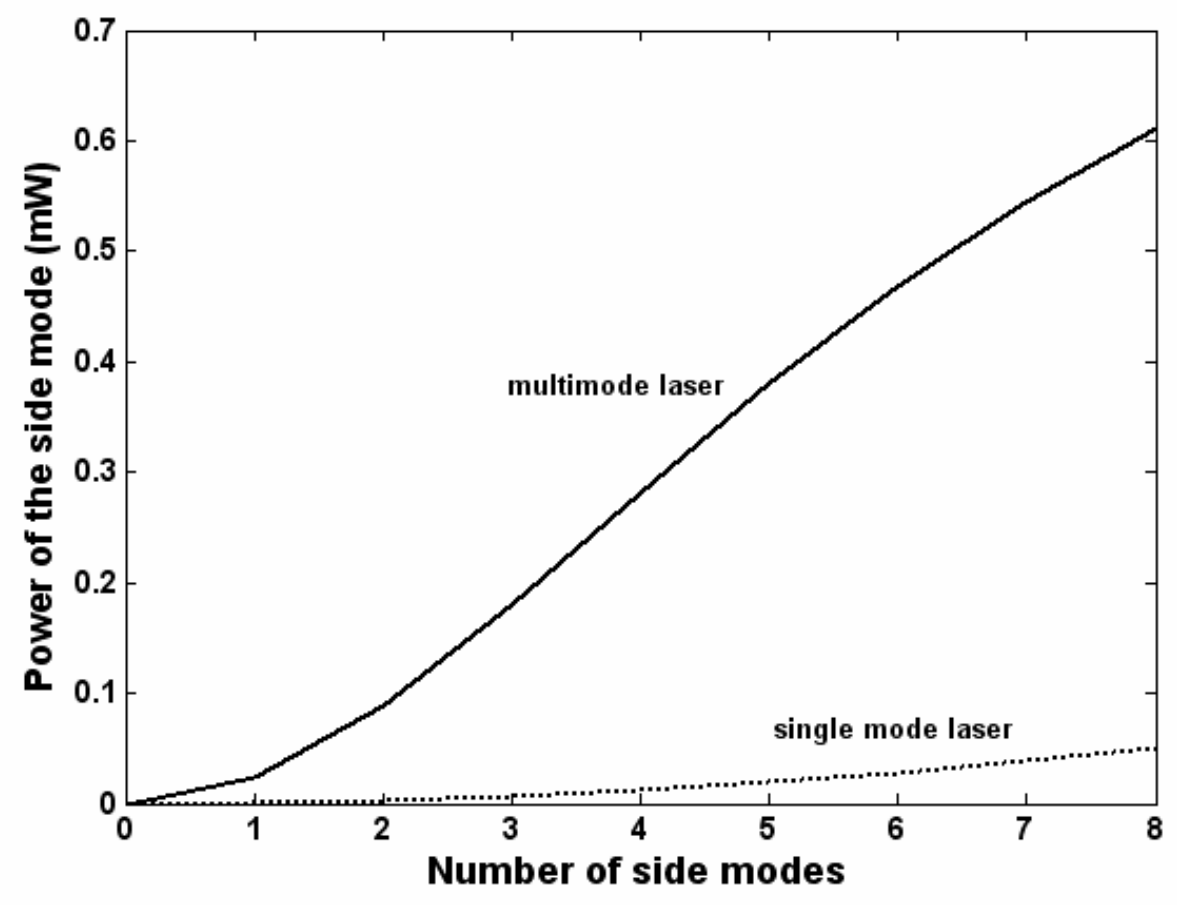

As can be seen from the figure, the power of the side mode for multimode laser increase linearly and sharply while for single mode laser the power of side mode increases very small with the number of modes.

\section{5- Conclusions:}

We can conclude from this study that optical network operation condition is mainly affected by the laser diode characteristics. The most effective parameter on the launched power of the transmitted signal is the number of modes in the output spectrum in the laser diode. The associated effects in addition to that are the MSR, RIN, and the chirp. These mentioned effects were studied through the normalized power output as a function of number of modes. High drop in power output will degrade signal to noise ratio and can be observed thoroughly in the eye diagram of the received signal. RIN value of $(-130 \mathrm{~dB} / \mathrm{Hz})$ showed better performance than $(-100 \mathrm{~dB} / \mathrm{Hz})$, making an attainable power in the fiber link. When chirping was taking into account a mode partition noise was observed degrading the signal to noise ratio. Generally speaking, laser diode parameters must be considered in order to make a liable optical network like using a DFB laser. 


\section{5- References:}

1) L. Dordová, Doctoral Degree Programme (1), FEEC BUT, E-mail: xdordo00@stud.feec.vutbr.cz.

2) W. C. Kwong, P. R. Prucnal, and M. C. Teich, "Coherent Subcarrier Fiber-optic Communication Systems with Phase-Noise Cancellation", IEEE Trans. Commun. 42, 16 (1994).

3) U. E. L. Christensen, and K. E. Stubkjzr, "Laser Linewidth Requirements and Improvements for Coherent Optical Beam Forming Networks in Satellite" J. Lightwave Techn. 9, 6 (1991).

4) A. Kaszubowska-Anandarajah, A. Mishra, A. Ellis, L. Barry, P. Anandarajah1, P. Perry, D. Reid1, J. O'Gorman, R. Phelan and B. Kelly," Cost Efficient Narrow Linewidth Laser Transmitter for Coherent Detection", (OSA/CLEO/QELS'08), San Jose, CThJJ3, May 2008.

5) E. I, Joseph, M.K. Fellow, and J. Hutchins, "Linewidth Measurements of MEMS-Based Tunable Lasers for Phase-Locking Applications", IEEE Photon. Techn. Lett. 17, 10 (2005).

6) G. H. Duan and P. Gallion, "Drive Current Noise Induced Linewidth in Tunable Multielectrode lasers", IEEE Photon. Techn. Lett. 3, 4 (1991).

7) S. Schneider, P. Borri, W. Langbein, U.e Woggon, R. L. Sellin, D. Ouyang, and D. Bimberg, "Linewidth Enhancement Factor in InGaAs Quantum-Dot Amplifiers", IEEE J. Quant. Elect. QE- 40, 10 (2004).

8) G. Licreol, "Recent Advances in Coherent Optical Communication", $\underline{\text { i@ creol.ucf.edu. }}$

9) T. Suhara, Semiconductor Laser Fundamentals, Marcel Dekker publisher, Osaka, Japan, 2004.

10) B. Moeyersoon, G. Morthier, M. Zhao, "Degradation of the Mode Suppression in Single-Mode Laser Diodes Due to Integrated Optical Amplifiers", IEEE J. Quant. Elect. QE- 40, 3 (2004).

11) G.P. Agrawal and N.K. Dutta, Semiconductor Laser, $2^{\text {nd }}$ Edition, Van Nostrand Reinhold, New York, N.Y. (1993).

12) K. Y. Lau, and A. Yariv, "Ultra-High Speed semiconductor Laser", J. Quantum Elect. 21, 121 (1995).

13) G.P. Agrawal, Fiber-Optic Communication System, $2^{\text {nd }}$ Edition. John Willy \& sons. Inc., N.Y., (1997).

14) C. J. S. DeCusatis, Fiber Optic Essentials, Academic Press, Burlington MA, USA, 2006.

15) H.F. Chen, J.M. Liu, and T.B. Simpson, "Response Characteristics of Direct Current Modulation on a Bandwidth Enhanced Semiconductor Laser Under Strong Injection locking", Opt. Commun. 173, 349 (2000). 